\title{
Inner-shell Absorption Lines of Fe VI- Fe XVI: A Many-body Perturbation Theory Approach
}

\author{
Ming F. Gu${ }^{1}$, Tomer Holczer ${ }^{2}$, Ehud Behar ${ }^{2}$, and Steven M. Kahn ${ }^{1}$
}

\begin{abstract}
We provide improved atomic calculation of wavelengths, oscillator strengths, and autoionization rates relevant to the $2 \rightarrow 3$ inner-shell transitions of Fe VIXVI, the so-called Fe M-shell unresolved transition array (UTA). A second order many-body perturbation theory is employed to obtain accurate transition wavelengths, which are systematically larger than previous theoretical results by 15-45 $\mathrm{m \AA}$. For a few transitions of Fe XVI and Fe XV where laboratory measurements exist, our new wavelengths are accurate to within a few $\mathrm{m} \AA$. Using these new calculations, the apparent discrepancy in the velocities between the Fe Mshell UTA and other highly ionized absorption lines in the outflow of NGC 3783 disappears. The oscillator strengths in our new calculation agree well with the previous theoretical data, while the new autoionization rates are significantly larger, especially for lower charge states. We attribute this discrepancy to the missing autoionization channels in the previous calculation. The increased autoionization rates may slightly affect the column density analysis of the Fe M-shell UTA for sources with high column density and very low turbulent broadening. The complete set of atomic data is provided as an electronic table.
\end{abstract}

Subject headings: atomic data, galaxies: active, techniques: spectroscopic

\section{Introduction}

Since the first astrophysical detection of $2 \rightarrow 3$ innershell absorption lines of Fe VII-XII in the X-ray spectrum of IRAS $13349+2438$ obtained with the Reflection Grating Spectrometer (RGS) on board XMM-Newton (Sako 2001), this so-called Fe M-shell unresolved transition array (UTA) has been identified in many soft X-ray sources. The UTA is mainly

\footnotetext{
${ }^{1}$ Kavli Institute for Particle Astrophysics and Cosmology and Department of Physics, Stanford University, CA 94305, USA

${ }^{2}$ Department of Physics, Technion, Haifa 32000, Israel
} 
comprised of a cluster of lines originating from $2 p-3 d$ transitions of M-shell iron ions that are located between 15-17 $\AA$. These lines, when properly modeled, provide important information on the ionization structure, column density, and outflow kinematics of the absorbing materials. Behar et al. (2001) calculated a complete set of atomic data for such modeling using the Hebrew University Lawrence Livermore Atomic Code (Bar-Shalom et al. 2001, HULLAC, , and provided an abbreviated list of transition wavelengths, oscillator strengths, and autoionization rates. That complete data set has also been incorporated in some commonly used plasma modeling codes.

In most observations where the Fe M-shell UTA has been identified, individual features from different ionization states are not resolved, mostly due to significant turbulent velocity broadening $\left(>700 \mathrm{~km} \mathrm{~s}^{-1}\right.$ ) at the source. Low statistics and insufficient spectral resolution may also hamper the identification of individual charge states. Therefore, one has to depend on global photoionized plasma models to constrain the physical conditions of the absorbing gas. In global fits, where individual UTAs are unresolved, the exact rest-frame position of each UTA is particularly important, especially since current photoionization balance calculations fail to predict the formation of Fe M-shell ions consistently with highly ionized species of other elements (Netzer 2003, 2004; Kraemer et al. 2004).

Recently, Holczer et al. (2005) have reexamined the Chandra High Energy Transmission Grating Spectrometer (HETGS) spectrum of the active galactic nucleus (AGN) of NGC 3783, by coadding all $900 \mathrm{ks}$ available observations. The low turbulent velocity of the outflow of NGC 3783, the high spectral resolution of the HETGS, and the very long exposure time enabled the individual absorption features from different Fe M-shell ions to be resolved. The analysis of Holczer et al. (2005) showed that the outflow velocity associated with the Fe M-shell UTA appears to be different from those of other highly-ionized species, namely, Fe XVII, O VII, and O VIII. In fact, the data prefer a zero velocity for the gas associated with Fe M-shell ions, and a $-590 \mathrm{~km} \mathrm{~s}^{-1}$ outflow velocity for the gas associated with more highly ionized ions. Therefore, a two component model was proposed where the Fe M-shell ions are not part of the AGN outflow, with an important implication of much lower mass loss rate.

As stated in Holczer et al. (2005), the conclusions of that paper depend strongly on the calculated HULLAC wavelengths. The velocities of the Fe M-shell UTA can be distinguished from those of other ions only if the HULLAC wavelengths are accurate to better than $30 \mathrm{~m} \AA$, which corresponds to a velocity of $\sim 590 \mathrm{~km} \mathrm{~s}^{-1}$ at $16 \mathrm{~m} \AA$. However, it has been shown that the theoretical method employed by HULLAC can generally produce un-

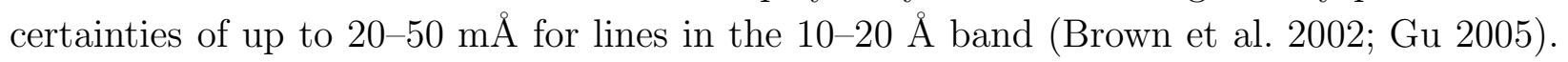
Unfortunately, no laboratory measurements exist for the wavelengths of relevant ions except 
for a few strong lines of Fe XVI and Fe XV (Brown et al. 2001). For these few lines, the HULLAC wavelengths are indeed underestimated by 20-30 mA. However, the strongest and cleanest absorption features in the observed spectrum of NGC 3783 are from Fe IX-XI. If the HULLAC wavelengths for these ions are also underestimated by similar amounts, the implied outflow velocity would be similar to that derived from Fe XVII and O ions. Holczer et al. (2005) argued that errors in the calculated wavelengths would be distributed randomly in both directions, and therefore, the systematic shift observed in the data is unlikely a result of theoretical wavelength errors. Furthermore, it was shown that the HULLAC errors for the strongest $2 \mathrm{p}-3 \mathrm{~d}$ transitions (those which determine the UTA centroid) of the Fe L-shell ions are generally much smaller than $30 \mathrm{~mA}$ (Brown et al. 2002; Holczer et al. 2005). The present work, however, shows that these indirect indications concerning the HULLAC accuracy for the Fe M-shell UTA wavelengths were misleading and that the assumption that these wavelengths could be used for velocity measurements was premature.

Recently, Gu (2005) showed that a combined configuration interaction and second order many-body perturbation theory (MBPT) is capable of predicting wavelengths of $2 \rightarrow 3$ transitions of Fe XVII-XXIV to within a few $\mathrm{m} \AA$. The $2 \rightarrow 3$ lines of the Fe M-shell UTA are similar to those of Fe XVII-XXIV, except for the addition of M-shell spectator electrons. Following the unexpected result of Holczer et al. (2005), in this paper, we apply an improved version of the MBPT method to the $2 \rightarrow 3$ transitions of Fe VI-XVI. The resulting wavelengths of the UTA lines are found to be systematically larger than HULLAC ones by 15-45 $\mathrm{m} \AA$. We also give improved autoionization rates, which are significantly larger than the previous HULLAC results. The new data are used to model the NGC 3783 spectrum, and the outflow velocity derived from Fe M-shell UTA is found to be consistent with that associated with Fe XVII and O VII-VIII ions.

In $\S 2$, we describe the detail of MBPT and its implementation. $\S 3$ presents the results of the calculation, and their comparison with the previous HULLAC data set. A brief summary is given in $\S 4$.

\section{Theoretical Method}

The present calculation is based on the Rayleigh-Schrödinger perturbation theory for

a multi-configurational model space. The brief description of the method presented here closely follows Lindgren (1974), and readers interested in a more detailed account should consult Lindgren (1974). 
The method tries to solve the Schrödinger equation

$$
H_{D C B} \Psi_{k}=E_{k} \Psi_{k}
$$

with perturbation expansion. The no-pair Dirac-Coulomb-Breit (DCB) Hamiltonian $H_{D C B}$ for an $N$-electron ionic system can be written as (Sucher 1980),

$$
H_{D C B}=\sum_{i}\left[h_{d}(i)-\frac{Z}{r_{i}}\right]+\sum_{i<j}\left(\frac{1}{r_{i j}}+B_{i j}\right)
$$

where $h_{d}(i)$ is the Dirac Hamiltonian for one free electron, $Z$ is the nuclear charge, $r_{i}$ is the radial coordinate of the electron $i, r_{i j}$ is the distance between the electrons $i$ and $j$, and $B_{i j}$ is the frequency-independent Breit interaction

$$
B_{i j}=-\frac{1}{2 r_{i j}}\left[\alpha_{\mathbf{i}} \cdot \alpha_{\mathbf{j}}+\frac{\left(\alpha_{\mathbf{i}} \cdot \mathbf{r}_{\mathbf{i j}}\right)\left(\alpha_{\mathbf{j}} \cdot \mathbf{r}_{\mathbf{i j}}\right)}{r_{i j}^{2}}\right],
$$

where $\alpha_{\mathbf{i}}$ is a matrix vector constructed from Pauli spin matrices $\sigma_{\mathbf{i}}$

$$
\alpha_{\mathbf{i}}=\left(\begin{array}{cc}
0 & \sigma_{\mathbf{i}} \\
\sigma_{\mathbf{i}} & 0
\end{array}\right)
$$

$H_{D C B}$ is split up into a model Hamiltonian $H_{0}$, and a perturbation $V$. In the present case, a convenient choice is

$$
\begin{aligned}
H_{0} & =\sum_{i}\left[h_{d}(i)+U\left(r_{i}\right)\right] \\
V & =-\sum_{i}\left[\frac{Z}{r_{i}}+U\left(r_{i}\right)\right]+\sum_{i<j}\left(\frac{1}{r_{i j}}+B_{i j}\right),
\end{aligned}
$$

where $U(r)$ is a model potential including the screening effects of all electrons, whose appropriate choice makes $V$ as small as possible. In many applications, $U(r)$ is taken to be the Hartree-Fock potential of a suitable configuration. Such a choice simplifies the many-body expansion because many terms in the series vanish exactly. However, Hartree-Fock potential is non-local and complicates the generation of the zero-th order radial wavefunctions. In the present implementation, $U(r)$ is approximated by a local central potential, and is derived from a Dirac-Fock-Slater self-consistent field calculation, which minimizes the weighted mean energy of all relevant configurations. As long as the perturbation potential $V$ is kept small enough, the exact choice for $U(r)$ does not affect the final results.

The eigenfunctions $\Phi_{k}$ and eigenvalues $E_{k}^{0}$ of $H_{0}$ are easily obtained by forming Slater determinants from single-electron wavefunctions once $U(r)$ is determined. A subset of $\Phi_{k}$ 
will define a model space $M$, and the remaining states belong to the orthogonal space $N$. A projection operator $P$ is defined for $M$, which produces a state in the model space when operated on an eigenfunction of the full Hamiltonian

$$
\Psi_{k}^{0}=P \Psi_{k}
$$

where $\Psi_{k}^{0}$ is generally a linear combination of the subset of $\Phi_{k}$ that belong to the model space $M$.

As described in Lindgren (1974), one can define a wave operator, $\Omega$, that transforms $\Psi_{k}^{0}$ back to $\Psi_{k}$,

$$
\Psi_{k}=\Omega \Psi_{k}^{0}
$$

The original Schrödinger equation can then be transformed to

$$
H_{e f f} \Psi_{k}^{0}=P H \Omega \Psi_{k}^{0}=E_{k} \Psi_{k}^{0}
$$

This equation defines an effective Hamiltonian in the model space

$$
H_{e f f}=P H_{0} P+P V \Omega
$$

whose eigenvalues are the true eigenenergies of the full Hamiltonian. The effective Hamiltonian is generally non-hermitian, and the eigenfunctions, $\Psi_{k}^{0}$ are not necessarily orthogonal. It follows from the Schrödinger equation and the definition of $\Omega$ that the wave operator satisfies

$$
\left[\Omega, H_{0}\right]=V \Omega-\Omega V \Omega,
$$

which is the starting point of the many-body expansion formula. The first order expansion of $P V \Omega$ can be written as

$$
<\Phi_{i}\left|V \Omega^{(1)}\right| \Phi_{j}>=<\Phi_{i}|V| \Phi_{j}>+\sum_{r \in N} \frac{<\Phi_{i}|V| \Phi_{r}><\Phi_{r}|V| \Phi_{j}>}{E_{j}^{0}-E_{r}^{0}}
$$

in terms of matrix elements in the model space $M$. The first order effective Hamiltonian is then

$$
<\Phi_{i}\left|H_{\text {eff }}^{(1)}\right| \Phi_{j}>=H_{D C B}^{i j}+\sum_{r \in N} \frac{V^{i r} V^{r j}}{E_{j}^{0}-E_{r}^{0}}
$$

where $H_{D C B}^{i j}=<\Phi_{i}\left|H_{D C B}\right| \Phi_{j}>$, and $V^{i r}=<\Phi_{i}|V| \Phi_{r}>$. By solving the generalized eigenvalue problem for the first order effective Hamiltonian, one obtains the eigenvalues in second order. 
The energy levels obtained in the present work are based on the solution of this generalized eigenvalue problem. This is slightly different from the method used in $\mathrm{Gu}$ (2005), where following Vilkas et al. (1999), the total second order energy is expressed as

$$
E_{k}=E_{k}^{C I}+E_{k}^{2}
$$

where $E_{k}^{C I}$ is the eigenvalues of $P H_{D C B} P$ in the model space with the corresponding eigenvectors $b_{k i}^{0}$, and $E_{k}^{2}$ is the second order correction due to correlations in the space $N$

$$
E_{k}^{2}=\sum_{r \in N} \sum_{i, j \in M} b_{k i}^{0} b_{k j}^{0} \frac{V^{i r} V^{r j}}{E_{j}^{0}-E_{r}^{0}} .
$$

This is in fact a good approximation of the generalized eigenvalue solution for $H_{e f f}^{(1)}$. If one replaces the eigenvectors $b_{k i}^{0}$ with the true eigenvectors $b_{k i}$ of $H_{e f f}^{(1)}$, the above expression becomes the exact eigenvalues of the first order effective Hamiltonian. The uncertainties introduced by using the eigenvectors of $P H_{D C B} P$ instead of $H_{\text {eff }}^{(1)}$ are generally of higher order, making it a valid approximation. Nonetheless, the more accurate method of solving the generalized eigenvalue problem for $H_{\text {eff }}^{(1)}$ is used in the present work.

In the present work, we restrict the calculation to the ground state energies of $1 s^{2} 2 l^{8} 3 l^{q}$ $(1 \leq q \leq 11)$ configurations, and the energies for states in the $1 s^{2} 2 l^{7} 3 l^{q+1}$ configuration that are connected to the ground state by electric dipole transitions. These are the absorption lines that appear in AGN where the ions are predominantly in the ground states. The model potential are calculated separately for the $1 s^{2} 2 l^{8} 3 l^{q}$ and $1 s^{2} 2 l^{7} 3 l^{q+1}$ configurations. The model space $M$ consists of these configurations, and the $N$ space consists of all single and double excitations from them. Because the $N$ space contains not only bound states, but also continuum, a finite basis set method is used to evaluate the perturbation expansion to convergence. The basis set is derived by imposing the boundary condition $Q\left(r_{b}\right) / P\left(r_{b}\right)=b$ for the single electron radial wavefunctions, where $Q(r)$ and $P(r)$ are the small and large components of the Dirac spinor, $r_{b}$ is the boundary radius, which is chosen to be large enough to contain the $1 s, 2 l$, and $3 l$ wavefunction amplitudes, and $b$ is an arbitrary constant chosen to be $\kappa / 2 r_{b} c$, where $\kappa$ is the relativistic angular momentum quantum number of the one-electron orbital, and $c$ is the speed of light. With this choice, the boundary condition reduces to $P^{\prime}\left(r_{b}\right) / P\left(r_{b}\right)=0$ in the non-relativistic limit.

Several small corrections to the Hamiltonian are also included in the calculations, namely, finite nuclear size, nuclear recoil, vacuum polarization, and electron self-energy. These are all taken into account with standard procedures of the atomic structure theory.

In addition to energies, we also calculate the oscillator strengths and radiative rates for $2 l-3 l^{\prime}$ transitions that are connected to the ground state of each ion. Because the $1 s^{2} 2 l^{7} 3 l^{q+1}$ 
states are highly autoionizing, the autoionization rates dominate the total lifetime of these levels. We calculate all possible autoionization channels between $1 s^{2} 2 l^{7} 3 l^{q+1}$ and $1 s^{2} 2 l^{8} 3 l^{q-1}$ configurations. In calculating the radiative and autoionization rates, one may either use the

eigenvectors $b_{k i}^{0}$ of $P H_{D C B} P$, or the generalized eigenvectors $b_{k i}$ of $H_{e f f}^{(1)}$. We have verified that the differences in the two choices are within $\sim 20 \%$ for the ions considered in the present work, and we adopt the results of the latter method, which is generally not very different from that of HULLAC.

For ions from Fe I-V, we have not carried out the MBPT calculation, since MBPT is expected to be less accurate for near neutral ions, and also because these ions are more complex. To give a complete database for the Fe M-shell UTA, we have made simple configuration interaction calculations for them, and present the results along with MBPT calculations for Fe VI-XVI.

\section{Results}

In Figure 1, we compare the wavelengths and absorption oscillator strengths of the present calculation and those of Behar et al. (2001) for Fe VII-XVI. As can be seen, the oscillator strengths in the two calculations agree well with each other except for a few isolated weak transitions. However, the wavelengths in the present work are systematically larger than the HULLAC results by 15-45 $\mathrm{m} \AA$ for some of the strongest lines. The differences between the oscillator strength weighted wavelengths in the two calculations are also shown in the figure. We have no simple explanation for why the MBPT wavelengths are systematically longer than the configuration-interaction method of HULLAC.

Table 1 gives the complete list of Fe M-shell UTA transitions with the ground states as the lower levels. The database is a combination of MBPT results for Fe VI-XVI and configuration interaction results for Fe $\mathrm{I}-\mathrm{V}$. The tabulated data consist of wavelengths, oscillator strengths, radiative transition rates, and autoionization rates. The autoionization rates are sums over all relevant autoionization channels. The radiative transition rates are those between the ground states and the specific upper levels, i.e., possible radiative decays to excited states are not included. The omission of these alternative radiative channels should have negligible effects on the total natural widths of the upper levels, since autoionization rates dominate the radiative rates in most cases, and for a few lines of Fe XVI and XV, where radiative rates are comparable to autoionization rates, decays to the ground states are the dominant branches.

In addition to wavelengths, another major difference between the present and the earlier 
calculations of Behar et al. (2001) is in the autoionization rates. The present autoionization rates are significantly larger than the previous results, especially for lower charge states. The discrepancy is caused by additional autoionization channels that are included here. Let us take the $1 s^{2} 2 s^{2} 2 p^{5} 3 s^{2} 3 p^{6} 3 d$ excited levels of Fe IX as an example. In Behar et al. (2001), only the autoionization channels that involve the ejection of the $3 \mathrm{~d}$ electron, i.e. toward $1 s^{2} 2 s^{2} 2 p^{6}(3 s 3 p)^{5}$, were considered. The present addition of channels to levels of $1 s^{2} 2 s^{2} 2 p^{6}(3 s 3 p)^{6} 3 d$ gives total autoionization rates that are $\sim 3.7$ times higher. The increased autoionization rates broaden further the natural line width and may have implications for the curve of growth if turbulent broadening is particularly small. On the other hand, if the source has significant turbulent broadening exceeding hundreds of $\mathrm{kms}^{-1}$, as is the usual case for AGN outflows, this effect is unlikely to be observed.

Holczer et al. (2005) recently reanalyzed the HETGS spectra of NGC 3783 in the Fe Mshell UTA region. The HULLAC data of Behar et al. (2001) was used in that analysis. The major conclusion was that the outflow velocity of the gas associated with Fe M-shell ions are significantly less than that derived from the Fe XVII, O VII, and O VIII lines. By comparing the deepest observed centroid of each ion and the HULLAC-calculated individual-ion centroid for Fe IX-XVI, velocities between -374 and $118 \mathrm{~km} \mathrm{~s}^{-1}$ were derived. The same comparison, but now carried out with the present MBPT data is shown in Table 2. Except for a few ions where the identification of a unique UTA centroid becomes difficult, the velocities range between -450 and $-780 \mathrm{~km} \mathrm{~s}^{-1}$. We have fitted a uniform outflow velocity $\left(-590 \mathrm{~km} \mathrm{~s}^{-1}\right)$ model to the same NGC 3783 spectrum of Holczer et al. (2005), using the present atomic data for Fe VI-XVI. The results are shown in Figure 2. Inspection of Figure 2 shows that no systematic discrepancies between the observed and theoretical absorption features for the Fe M-shell UTA can be readily identified, and therefore, the Fe M-shell and other more ionized ions appear to belong to the same kinematic system.

We have been able to identify one single laboratory measurement of Fe XVI lines against which we may check the accuracy of the present calculation. Brown et al. (2001) gave the wavelengths of the three strongest $2 p-3 d$ transitions identified in the emission spectrum taken at the Lawrence Livermore National Laboratory's electron beam ion trap. One of the three lines is blended with a strong Fe XVII line, and the other two lines are unblended, which allowed the accurate determination of their wavelengths. The two lines are $2 p^{6} 3 s(J=1 / 2)-$ $2 p_{1 / 2} 2 p_{3 / 2}^{4} 3 s 3 d_{3 / 2}(J=1 / 2,3 / 2)$ transitions, and have measured wavelengths of $15.208(4)$ and 15.115(6) $\AA$. The present work gives wavelengths of 15.2086 and $15.1127 \AA$ respectively, which agree very well with the measured values. In the contrary, Behar et al. (2001) gave wavelengths of 15.1890 and $15.0762 \AA$ respectively, which are 20-40 $\mathrm{m} \AA$ too small. Brown et al. (2001) also identified the strongest Fe XV line, $2 p^{6} 3 s^{2}(J=0)-2 p_{1 / 2} 2 p_{3 / 2}^{4} 3 s^{2} 3 d_{3 / 2}(J=$ 1 ), in their spectrum, but did not give its measured wavelength. Through inspection of 
Figure 1 in their paper and private communications, we estimate that its wavelength is 15.353(5) $\AA$, which also agrees reasonably well with the present result of $15.3588 \AA$, but $37 \mathrm{~m} \AA$ larger than the HULLAC result. Unfortunately, the Fe XV and XVI absorption lines are relatively weak in the NGC 3783 spectrum, and Fe XVI lines are severely blended with Fe XVII lines. Nevertheless, the excellent agreement between the present results and the measurement for these few lines lends credibility to the MBPT treatment of the entire iso-nuclear sequence.

\section{Conclusions}

We have developed a second order many-body perturbation theory with multi-reference model space. We apply the method to the calculation of wavelengths, oscillator strengths, and autoionization rates of $\mathrm{Fe} \mathrm{M}$-shell UTA arising from $2 p-3 d$ transitions originating from the ground state of Fe VI-XVI. The wavelengths obtained in the present work are system-

atically larger than the HULLAC calculation of Behar et al. (2001); the present oscillator strengths of the strong absorption lines agree well with the HULLAC results; and the autoionization rates of Behar et al. (2001) are found to be missing important autoionization channels, especially for lower charge states. Using the present data for Fe VI-XVI, we find no evidence that the outflow velocity of the gas associated with Fe M-shell ions is different from that derived from Fe XVII and O VII-VIII ions, as claimed in the recent analysis of Holczer et al. (2005) using the HULLAC data. A complete list of Fe M-shell UTA lines are given, which include the present MBPT calculation for Fe VI-XVI, and a simple configuration interaction calculation for Fe I-V. We recommend that this new database should be preferred over the earlier HULLAC calculation of Behar et al. (2001) in the analyses of future absorption spectroscopy where Fe M-shell UTA is prominent.

M.F. Gu and S.M. Kahn acknowledges the partial support of NASA grants NAG5-5419 and NNGG04GL76G. The research at the Technion was supported by The Israel Science Foundation (grant no. 28/03). E. Behar thanks the Stanford group for their hospitality during a visit in July 2005. We thank Shai Kaspi for making the 900 ks HETGS integrated spectrum available to us.

\section{REFERENCES}

Bar-Shalom A., Klapisch, M., \& Oreg, J. 2001, J. Quant. Spec. Radiat. Transf., 71, 169

Behar, E., Sako, M., \& Kahn, S. M. 2001, ApJ, 563, 497 
Brown, G. V., Beiersdorfer, P., Chen, H., \& Reed, K. J. 2001, ApJ, 557, L78

Brown, G. V., Beiersdorfer, P., Liedahl, D. A., Widmann, K., Kahn, S. M., \& Clothiaux, E. J. 2002, ApJS, 140, 589

Gu, M. F. 2005, ApJS, 156, 105

Holczer, T., Behar, E., \& Kaspi, S. 2005, ApJ, in press, astro-ph/0507027

Kaspi, S., Netzer, H., Chelouche, D., George, I. M., Nandra, K., \& Turner, T. J. 2004, ApJ, 611

Kraemer, S. B., Ferland, G. J., \& Gabel, J. R. 2004, ApJ, 604, 556

Lindgren, I. 1974, J. Phys. B, 7, 2441

Netzer, H. 2003, ApJ, 599, 933

—. 2004, ApJ, 604, 551

Sako, M. 2001, A\&A, 365, L168

Sucher, J. 1980, Phys. Rev. A, 22, 348

Vilkas, M. J., Ishikawa, Y., \& Koc, K. 1999, Phys. Rev. A, 60, 2808 
Table 1. Wavelengths, oscillator strengths, radiative decay rates, and autoionization rates of $2 \rightarrow 3$ transitions from the ground states of Fe I-XVI. For Fe VI-XVI, the MBPT method is used and for Fe I-VI, the standard configuration interaction method is used.

\begin{tabular}{|c|c|c|c|c|c|c|c|}
\hline Ion & Upper & $\lambda(\AA)$ & $A^{r}\left(\mathrm{~s}^{-1}\right)$ & $A^{a}\left(\mathrm{~s}^{-1}\right)$ & $A^{a}\left(\mathrm{~s}^{-1}\right)^{\mathrm{a}}$ & $f_{i j}$ & Configuration $(J)^{\mathrm{b}}$ \\
\hline XVI & 25 & 15.2652 & $1.41 \mathrm{E}+13$ & $2.11 \mathrm{E}+13$ & $2.63 \mathrm{E}+13$ & $9.88 \mathrm{E}-01$ & $2 p_{1 / 2} 3 s_{1 / 2} 3 d_{5 / 2}\left(J=\frac{3}{2}\right)$ \\
\hline XVI & 26 & 15.2086 & $2.38 \mathrm{E}+13$ & $1.68 \mathrm{E}+10$ & $1.39 \mathrm{E}+13$ & $8.27 \mathrm{E}-01$ & $2 p_{1 / 2} 3 s_{1 / 2} 3 d_{3 / 2}\left(J=\frac{1}{2}\right)$ \\
\hline XVI & 27 & 15.1127 & $8.92 \mathrm{E}+12$ & $4.03 \mathrm{E}+13$ & $6.33 \mathrm{E}+13$ & $6.11 \mathrm{E}-01$ & $2 p_{1 / 2} 3 s_{1 / 2} 3 d_{3 / 2}\left(J=\frac{\tilde{3}}{2}\right)$ \\
\hline XV & 16 & 15.3588 & $1.78 \mathrm{E}+13$ & $3.76 \mathrm{E}+13$ & $4.27 \mathrm{E}+13$ & $1.89 \mathrm{E}+00$ & $2 p_{1 / 2} 3 d_{3 / 2}(J=1)$ \\
\hline $\mathrm{XV}$ & 14 & 15.5965 & $7.64 \mathrm{E}+12$ & $4.57 \mathrm{E}+13$ & $6.93 \mathrm{E}+13$ & $8.36 \mathrm{E}-01$ & $2 p_{3 / 2}^{3} 3 d_{5 / 2}(J=1)$ \\
\hline XV & 18 & 14.1828 & $3.06 \mathrm{E}+12$ & $1.10 \mathrm{E}+14$ & $3.24 \mathrm{E}+14$ & $2.77 \mathrm{E}-01$ & $2 s_{1 / 2} 3 p_{3 / 2}(J=1)$ \\
\hline XIV & 51 & 15.6339 & $6.11 \mathrm{E}+12$ & $4.51 \mathrm{E}+13$ & $3.58 \mathrm{E}+13$ & $4.48 \mathrm{E}-01$ & $2 p_{1 / 2} 3 p_{1 / 2} 3 d_{3 / 2}\left(J=\frac{3}{2}\right)$ \\
\hline XIV & 55 & 15.5555 & $1.16 \mathrm{E}+13$ & $9.90 \mathrm{E}+13$ & $8.53 \mathrm{E}+13$ & $4.22 \mathrm{E}-01$ & $2 p_{1 / 2} 3 p_{1 / 2} 3 d_{3 / 2}\left(J=\frac{1}{2}\right)$ \\
\hline XIV & 53 & 15.6098 & $5.06 \mathrm{E}+12$ & $4.88 \mathrm{E}+13$ & $9.43 \mathrm{E}+13$ & $3.70 \mathrm{E}-01$ & $2 p_{1 / 2} 3 p_{3 / 2} 3 d_{3 / 2}\left(J=\frac{3}{2}\right)$ \\
\hline XIII & 31 & 15.8898 & $4.87 \mathrm{E}+12$ & $1.76 \mathrm{E}+14$ & $2.14 \mathrm{E}+14$ & $5.53 \mathrm{E}-01$ & $2 p_{3 / 2}^{3} 3 p_{1 / 2} 3 p_{3 / 2} 3 d_{5 / 2}(J=1)$ \\
\hline XIII & 33 & 15.8495 & $4.13 \mathrm{E}+12$ & $1.01 \mathrm{E}+14$ & $1.30 \mathrm{E}+14$ & $4.67 \mathrm{E}-01$ & $2 p_{3 / 2}^{3} 3 d_{5 / 2}(J=1)$ \\
\hline XIII & 38 & 15.7811 & $2.31 \mathrm{E}+12$ & $2.14 \mathrm{E}+14$ & $1.32 \mathrm{E}+14$ & $2.59 \mathrm{E}-01$ & $2 p_{1 / 2} 3 p_{1 / 2} 3 p_{3 / 2} 3 d_{3 / 2}(J=1)$ \\
\hline XII & 95 & 16.0253 & $4.06 \mathrm{E}+12$ & $1.19 \mathrm{E}+14$ & $1.26 \mathrm{E}+14$ & $2.35 \mathrm{E}-01$ & $2 p_{3 / 2}^{3} 3 p_{1 / 2} 3 p_{3 / 2}^{2} 3 d_{5 / 2}\left(J=\frac{5}{2}\right)$ \\
\hline XII & 97 & 16.0151 & $4.77 \mathrm{E}+12$ & $5.39 \mathrm{E}+14$ & $1.04 \mathrm{E}+14$ & $1.84 \mathrm{E}-01$ & $2 p_{1 / 2} 3 p_{3 / 2} 3 d_{3 / 2}\left(J=\frac{3}{2}\right)$ \\
\hline XII & 93 & 16.0345 & $9.30 \mathrm{E}+12$ & $2.08 \mathrm{E}+14$ & $1.01 \mathrm{E}+14$ & $1.79 \mathrm{E}-01$ & $2 p_{3 / 2}^{3} 3 p_{3 / 2}^{3} 3 d_{5 / 2}\left(J=\frac{1}{2}\right)$ \\
\hline $\mathrm{XI}$ & 90 & 16.1761 & $5.21 \mathrm{E}+12$ & $3.08 \mathrm{E}+14$ & $1.00 \mathrm{E}+14$ & $2.86 \mathrm{E}-01$ & $2 p_{1 / 2} 3 p_{3 / 2}^{2} 3 d_{3 / 2}(J=3)$ \\
\hline $\mathrm{XI}$ & 89 & 16.1788 & $8.63 \mathrm{E}+12$ & $2.98 \mathrm{E}+14$ & $9.94 \mathrm{E}+13$ & $2.03 \mathrm{E}-01$ & $2 p_{3 / 2}^{3} 3 p_{1 / 2} 3 p_{3 / 2}^{3} 3 d_{5 / 2}(J=1)$ \\
\hline XI & 74 & 16.2578 & $3.36 \mathrm{E}+12$ & $3.07 \mathrm{E}+14$ & $1.35 \mathrm{E}+14$ & $1.86 \mathrm{E}-01$ & $2 p_{1 / 2} 3 p_{1 / 2} 3 p_{3 / 2}^{3} 3 d_{3 / 2}(J=3)$ \\
\hline $\mathrm{X}$ & 39 & 16.3581 & $8.48 \mathrm{E}+12$ & $3.75 \mathrm{E}+14$ & $1.42 \mathrm{E}+14$ & $3.40 \mathrm{E}-01$ & $2 p_{1 / 2} 3 p_{3 / 2}^{3} 3 d_{3 / 2}\left(J=\frac{3}{2}\right)$ \\
\hline $\mathrm{X}$ & 38 & 16.3661 & $5.59 \mathrm{E}+12$ & $4.39 \mathrm{E}+14$ & $1.90 \mathrm{E}+14$ & $3.37 \mathrm{E}-01$ & $2 p_{1 / 2} 3 p_{3 / 2}^{3} 3 d_{3 / 2}\left(J=\frac{5}{2}\right)$ \\
\hline $\mathrm{X}$ & 43 & 16.2951 & $3.47 \mathrm{E}+12$ & $7.50 \mathrm{E}+14$ & $1.99 \mathrm{E}+14$ & $2.07 \mathrm{E}-01$ & $2 p_{3 / 2}^{3} 3 p_{3 / 2}^{3} 3 d_{5 / 2}\left(J=\frac{5}{2}\right)$ \\
\hline IX & 3 & 16.5392 & $1.29 \mathrm{E}+13$ & $6.31 \mathrm{E}+14$ & $1.72 \mathrm{E}+14$ & $1.59 \mathrm{E}+00$ & $2 p_{1 / 2} 3 d_{3 / 2}(J=1)$ \\
\hline IX & 2 & 16.7894 & $6.93 \mathrm{E}+12$ & $5.85 \mathrm{E}+14$ & $9.61 \mathrm{E}+13$ & $8.79 \mathrm{E}-01$ & $2 p_{3 / 2}^{3} 3 d_{5 / 2}(J=1)$ \\
\hline IX & 1 & 16.9464 & $8.44 \mathrm{E}+10$ & $5.57 \mathrm{E}+14$ & $6.26 \mathrm{E}+13$ & $1.09 \mathrm{E}-02$ & $2 p_{3 / 2}^{3} 3 d_{3 / 2}(J=1)$ \\
\hline VIII & 27 & 16.6784 & $1.03 \mathrm{E}+13$ & $6.37 \mathrm{E}+14$ & $2.16 \mathrm{E}+14$ & $4.30 \mathrm{E}-01$ & $2 p_{1 / 2} 3 d_{3 / 2}^{2}\left(J=\frac{3}{2}\right)$ \\
\hline VIII & 16 & 16.9289 & $4.72 \mathrm{E}+12$ & $6.42 \mathrm{E}+14$ & $1.97 \mathrm{E}+14$ & $3.04 \mathrm{E}-01$ & $2 p_{3 / 2}^{3} 3 d_{3 / 2} 3 d_{5 / 2}\left(J=\frac{5}{2}\right)$ \\
\hline VIII & 19 & 16.8030 & $3.38 \mathrm{E}+12$ & $6.01 \mathrm{E}+14$ & $1.64 \mathrm{E}+14$ & $2.14 \mathrm{E}-01$ & $2 p_{1 / 2} 3 d_{3 / 2} 3 d_{5 / 2}\left(J=\frac{5}{2}\right)$ \\
\hline VII & 46 & 16.9404 & $4.03 \mathrm{E}+12$ & $6.26 \mathrm{E}+14$ & $2.42 \mathrm{E}+14$ & $1.73 \mathrm{E}-01$ & $2 p_{1 / 2} 3 d_{3 / 2}^{2} 3 d_{5 / 2}(J=2)$ \\
\hline VII & 26 & 17.1276 & $2.52 \mathrm{E}+12$ & $7.69 \mathrm{E}+14$ & $3.74 \mathrm{E}+14$ & $1.55 \mathrm{E}-01$ & $2 p_{3 / 2}^{3} 3 d_{3 / 2} 3 d_{5 / 2}^{2}(J=3)$ \\
\hline VII & 25 & 17.1289 & $3.45 \mathrm{E}+12$ & $6.13 \mathrm{E}+14$ & $2.90 \mathrm{E}+14$ & $1.52 \mathrm{E}-01$ & $2 p_{3 / 2}^{3} 3 d_{3 / 2}^{2} 3 d_{5 / 2}(J=2)$ \\
\hline VI & 24 & 17.2656 & $3.80 \mathrm{E}+12$ & $7.30 \mathrm{E}+14$ & $3.88 \mathrm{E}+14$ & $2.55 \mathrm{E}-01$ & $2 p_{3 / 2}^{3} 3 d_{3 / 2}^{2} 3 d_{5 / 2}^{2}\left(J=\frac{5}{2}\right)$ \\
\hline VI & 35 & 17.2105 & $3.31 \mathrm{E}+12$ & $5.93 \mathrm{E}+14$ & $3.13 \mathrm{E}+14$ & $1.47 \mathrm{E}-01$ & $2 p_{3 / 2}^{3} 3 d_{3 / 2} 3 d_{5 / 2}^{3}\left(J=\frac{3}{2}\right)$ \\
\hline VI & 39 & 17.1881 & $2.72 \mathrm{E}+12$ & $7.06 \mathrm{E}+14$ & $2.33 \mathrm{E}+14$ & $1.21 \mathrm{E}-01$ & $2 p_{3 / 2}^{3} 3 d_{3 / 2}^{2} 3 d_{5 / 2}^{2}\left(J=\frac{3}{2}\right)$ \\
\hline
\end{tabular}

aTotal autoionization rates in the HULLAC calculation of Behar et al. (2001). This column is not present in the electronic version of this table, and is only included here to illustrated the difference between the present and HULLAC results.

${ }^{\mathrm{b}}$ The configurations are given in the $j j$ coupling notation, and closed subshells are omitted to save space.

Note. - Only three strongest absorption lines per ion from Fe VI-XVI are shown here. The entire table is included in the electronic version of the paper as a machine readable file. 
Table 2. Best-fit velocities and column densities for ions detected in the 14.9-17.5 region of the HETGS spectrum of NGC3783.

\begin{tabular}{|c|c|c|c|c|c|c|}
\hline Ion & $\begin{array}{l}\lambda_{\text {Observed }} \\
(\AA)\end{array}$ & $\begin{array}{l}\lambda_{\text {Rest }^{\mathrm{a}}}(\AA) \\
(\AA)\end{array}$ & $\begin{array}{l}\lambda_{\text {Model }}{ }^{\mathrm{b}} \\
(\AA)\end{array}$ & $\begin{array}{l}\text { Outflow Velocity }{ }^{\mathrm{c}} \\
\qquad\left(\mathrm{km} \mathrm{s}^{-1}\right)\end{array}$ & $\begin{array}{l}\text { Ion Column Density } \\
\quad\left(10^{16} \mathrm{~cm}^{-2}\right)\end{array}$ & Configuration $(J)$ \\
\hline \multirow[t]{2}{*}{$\mathrm{O}^{+7}$} & $15.144 \pm 0.003^{\mathrm{d}}$ & 15.176 & 15.146 & $-631 \pm 59$ & \multirow[t]{2}{*}{$400 \pm 60$} & $4 p(J=1 / 2,3 / 2)$ \\
\hline & $15.970 \pm 0.005^{\mathrm{e}}$ & 16.006 & 15.977 & $-675 \pm 94$ & & $3 p(J=1 / 2,3 / 2)$ \\
\hline \multirow[t]{3}{*}{$\mathrm{O}^{+6}$} & $17.351 \pm 0.005$ & 17.395 & 17.361 & $-759 \pm 86$ & \multirow[t]{3}{*}{$110 \pm 20$} & $1 s 5 p(J=1)$ \\
\hline & $17.161 \pm 0.005$ & 17.199 & 17.165 & $-663 \pm 87$ & & $1 s 6 p(J=1)$ \\
\hline & $17.048 \pm 0.005$ & 17.084 & 17.050 & $-632 \pm 88$ & & $1 s 7 p(J=1)$ \\
\hline \multirow{2}{*}{$\mathrm{Fe}^{+16}$} & $14.980 \pm 0.003$ & 15.013 & 14.985 & $-659 \pm 60$ & \multirow[t]{2}{*}{$3.0 \pm 0.5$} & $2 p_{1 / 2} 3 d_{3 / 2}(J=1)$ \\
\hline & $15.231 \pm 0.002^{\mathrm{f}}$ & 15.261 & 15.232 & $-590 \pm 39$ & & $2 p_{3 / 2} 3 d_{5 / 2}(J=1)$ \\
\hline $\mathrm{Fe}^{+15}$ & $15.231 \pm 0.002^{\mathrm{f}}$ & 15.265 & 15.232 & $-668 \pm 39$ & $1.6 \pm 0.4$ & $2 p_{1 / 2} 3 s_{1 / 2} 3 d_{5 / 2}\left(J=\frac{3}{2}\right)$ \\
\hline $\mathrm{Fe}^{+14}$ & $15.322 \pm 0.006$ & 15.359 & 15.329 & $-723 \pm 117$ & $0.4 \pm 0.1$ & $2 p_{1 / 2} 3 d_{3 / 2}(J=1)$ \\
\hline $\mathrm{Fe}^{+13}$ & $15.569 \pm 0.008^{\mathrm{g}}$ & 15.634 & 15.603 & $-1247 \pm 154$ & $1.1 \pm 0.4$ & $2 p_{1 / 2} 3 p_{1 / 2} 3 d_{3 / 2}\left(J=\frac{3}{2}\right)$ \\
\hline $\mathrm{Fe}^{+12}$ & $15.844 \pm 0.014 \mathrm{~g}$ & 15.890 & 15.816 & $-868 \pm 264$ & $1.2 \pm 0.3$ & $2 p_{3 / 2}^{3} 3 p_{1 / 2} 3 p_{3 / 2} 3 d_{5 / 2}(J=1)$ \\
\hline $\mathrm{Fe}^{+11}$ & $15.970 \pm 0.005^{\mathrm{e}}$ & 16.024 & 15.977 & $-1011 \pm 94$ & $2.3 \pm 1.5$ & $2 p_{3 / 2}^{3} 3 p_{1 / 2} 3 p_{3 / 2}^{2} 3 d_{5 / 2}\left(J=\frac{5}{2}\right)$ \\
\hline $\mathrm{Fe}^{+10}$ & $16.154 \pm 0.005$ & 16.179 & 16.150 & $-464 \pm 93$ & $3.0 \pm 1.2$ & $2 p_{1 / 2} 3 p_{3 / 2}^{2} 3 d_{3 / 2}(J=3)$ \\
\hline $\mathrm{Fe}^{+9}$ & $16.329 \pm 0.005$ & 16.360 & 16.327 & $-568 \pm 92$ & $5.5 \pm 1.0$ & $2 p_{1 / 2} 3 p_{3 / 2}^{3} 3 d_{3 / 2}\left(J=\frac{3}{2}\right)$ \\
\hline $\mathrm{Fe}^{+8}$ & $16.496 \pm 0.004$ & 16.539 & 16.507 & $-780 \pm 73$ & $4.0 \pm 0.5$ & $2 p_{1 / 2} 3 d_{3 / 2}(J=1)$ \\
\hline $\mathrm{Fe}^{+7 \mathrm{~h}}$ & $\cdots$ & 16.678 & $\cdots$ & $\cdots$ & $3.0 \pm 1.0$ & $2 p_{1 / 2} 3 d_{3 / 2}^{2}\left(J=\frac{3}{2}\right)$ \\
\hline $\mathrm{Fe}^{+6 \mathrm{~h}}$ & $\cdots$ & 17.129 & $\cdots$ & $\cdots$ & $2.0 \pm 0.7$ & $2 p_{1 / 2} 3 d_{3 / 2}^{2} 3 d_{5 / 2}(J=2)$ \\
\hline $\mathrm{Fe}^{+5 \mathrm{~h}}$ & $\cdots$ & 17.266 & $\cdots$ & $\cdots$ & $1.5 \pm 0.6$ & $2 p_{3 / 2}^{3} 3 d_{3 / 2}^{2} 3 d_{5 / 2}^{2}\left(J=\frac{5}{2}\right)$ \\
\hline $\mathrm{Fe}^{+4 \mathrm{~h}}$ & $\cdots$ & 17.328 & $\cdots$ & $\cdots$ & $1.5 \pm 0.6$ & $2 p_{3 / 2}^{3} 3 d_{3 / 2}^{2} 3 d_{5 / 2}^{3}(J=1)$ \\
\hline $\mathrm{Fe}^{+3 \mathrm{~h}}$ & $\cdots$ & 17.387 & $\cdots$ & $\cdots$ & $\leq 0.8$ & $2 p_{3 / 2}^{3} 3 d_{3 / 2}^{2} 3 d_{5 / 2}^{4}\left(J=\frac{3}{2}\right)$ \\
\hline
\end{tabular}

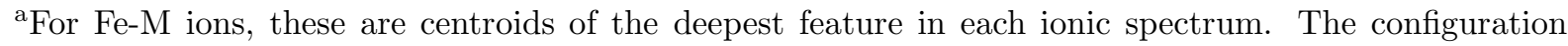
labels given in the last column also correspond to these deepest features.

${ }^{\mathrm{b}}$ Centroid in full multi-ion model (Fig. 2).

${ }^{\mathrm{c}}$ Estimated by $\left(\lambda_{\mathrm{Observed}}-\lambda_{\text {Rest }}\right) c / \lambda_{\text {Rest }}$. Errors reflect $90 \%$ confidence intervals.

${ }^{\mathrm{d}}$ Blend of $\mathrm{O}^{+7}$ and $\mathrm{Fe}^{+15}$ at $15.19 \AA$ fitted for two distinct features.

${ }^{\mathrm{e}}$ Unresolved blend of $\mathrm{O}^{+7}$ and $\mathrm{Fe}^{+11}$ reflected in the high velocity and large error on the $\mathrm{Fe}^{+11}$ column density.

${ }^{\mathrm{f}}$ Unresolved blend of $\mathrm{Fe}^{+16}$ and $\mathrm{Fe}^{+15}$.

${ }^{\text {g}}$ These UTAs have multiple minima making centroid determination difficult. (See Fig. 2.)

${ }^{\mathrm{h}}$ Indirect identification based on best-fit model; Strongly blended with the high- $n$ lines of $\mathrm{O}^{+6}$. 


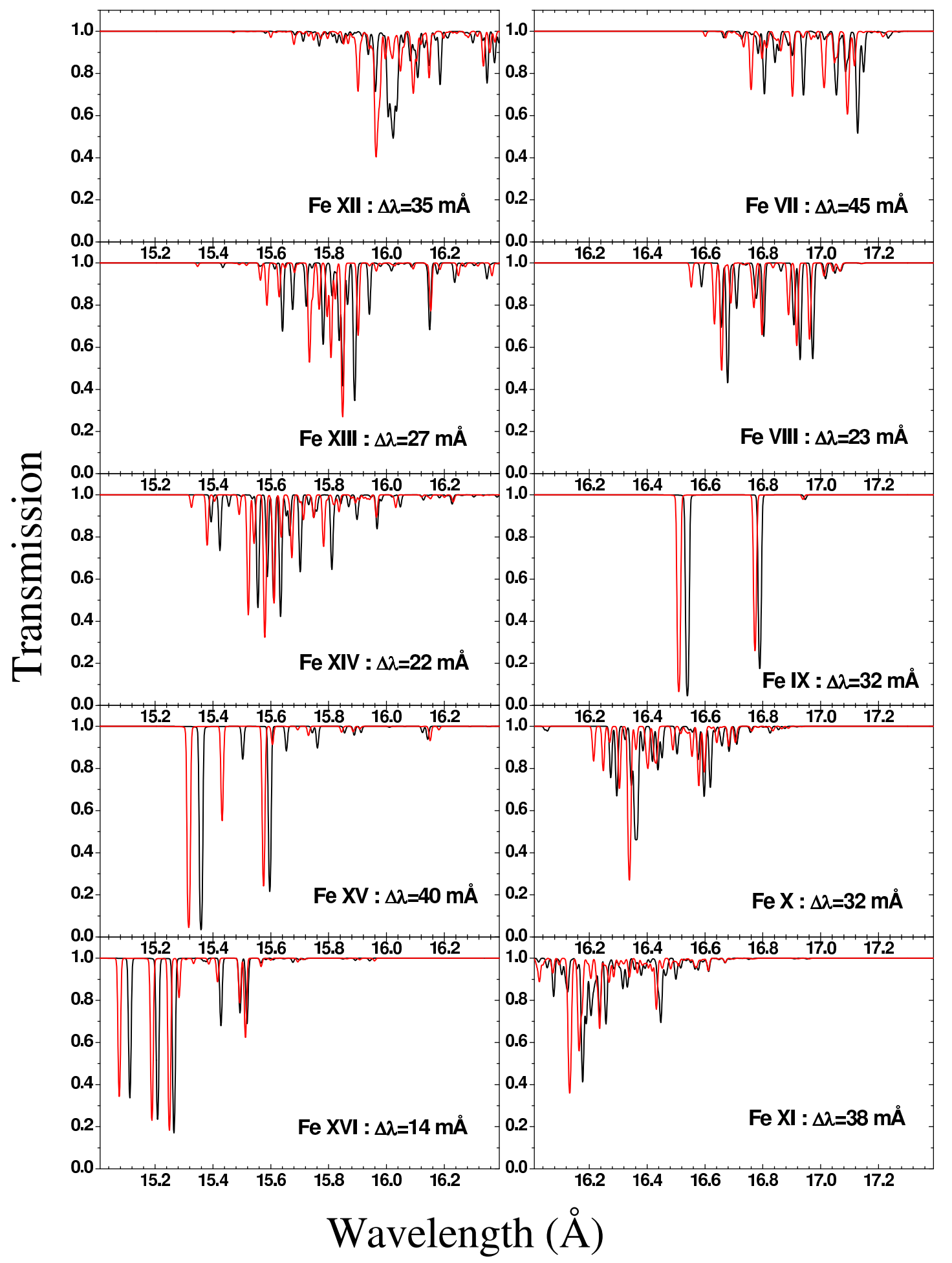

Fig. 1.- Absorption spectra of $2 \rightarrow 3$ transitions from the ground state of Fe VII-XVI at an arbitrary column density and turbulent velocity of, respectively, $8 \times 10^{15} \mathrm{~cm}^{-2}$ and $100 \mathrm{~km} \mathrm{~s}^{-1}$. Black lines are the present results and red lines are the HULLAC calculation of Behar et al. (2001). The value of $\Delta \lambda$ shown in each panel is the difference between the oscillator strength weighted wavelengths of the present and HULLAC calculations. 


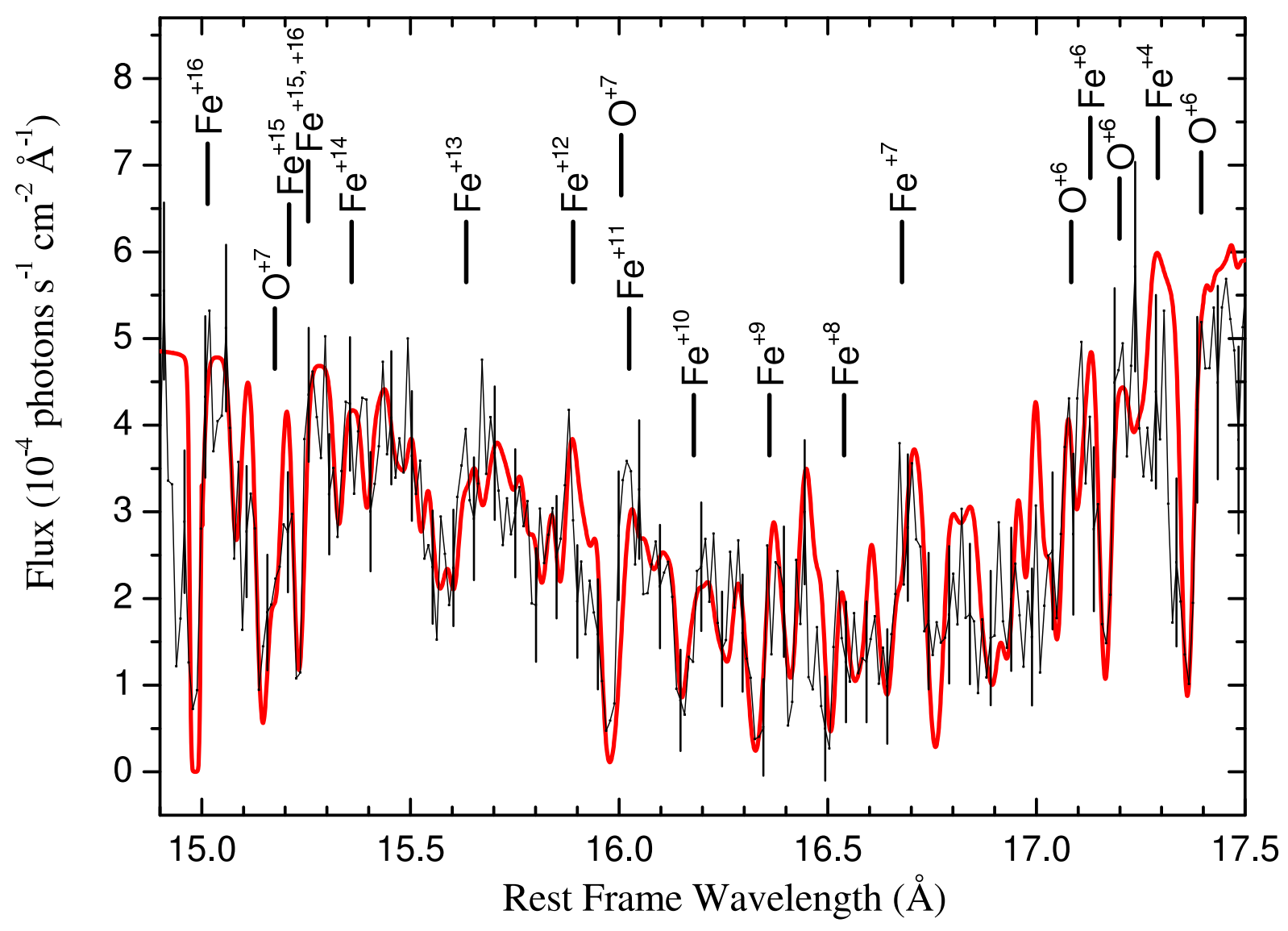

Fig. 2.- Chandra HETGS spectra of NGC 3783 in the Fe M-shell UTA region. Black line with error bars (plotted every six data points) are the data, red line is the model with a uniform outflow velocity of $-590 \mathrm{~km} \mathrm{~s}^{-1}$ using the present atomic data for Fe VI-XVI. 